\title{
A Proportional-Integral-Derivative Control Scheme of Mobile Robotic platforms using MATLAB
}

\author{
Kunal Agarwal $^{1}$, Shadan Mahtab ${ }^{2}$, Sourav Bandyopadhyay ${ }^{3}$, \\ Sauvik Das Gupta ${ }^{4}$ \\ ${ }^{1,2,3}$ Department of AEIE, Heritage Institute of Technology, Kolkata, West Bengal, India \\ ${ }^{4}$ School of Electrical \& Computer Engineering, Oklahoma State University, Stillwater, OK, USA
}

\begin{abstract}
This paper proposes an approach for the navigation of mobile robots namely Khepera 3 and iRobot Create in a designated environment. In this study, the navigation system of Khepera 3 includes the motion of the mobile robot at a particular angle to reach a particular goal and avoidance of the obstacle that may occur in its path. Obstacle avoidance is realized using Blending and Hard switching techniques respectively and the navigation system of iRobot Create includes its movement in a rectangular path. In controlling the navigation of the robots, Proportional-Integral-Derivative (PID) controller is used. The most suitable set of values of PID parameters implemented for safe and effective navigation of the robots have been presented. Computer simulation is done using MATLAB software.
\end{abstract}

Keywords: Navigation, Mobile robots, Obstacle avoidance, Blending, Hard switching, PID.

\section{Introduction}

A proportional-integral-derivative controller (PID controller) is a generic control loop feedback mechanism widely used in industrial control systems [1]. PID algorithm consists of three basic coefficients; Proportional, Integral and Derivative which are varied to get optimal response. The basic idea behind a PID controller is to read a sensor, then compute the desired actuator output by calculating Proportional, Integral, and Derivative responses and summing those three components to compute the output[2].

\section{a.Proportional Response}

\section{PID Theory}

The Proportional term produces an output value that is proportional to the current error value. The proportional response can be adjusted by multiplying the error by a constant called the proportional gain constant $\left(\mathrm{K}_{\mathrm{P}}\right)$.

The proportional response is given by:

$\mathrm{P}_{\text {out }}=\mathrm{K}_{\mathrm{P}} * \mathrm{e}(\mathrm{t})$

Where, $\mathrm{K}_{\mathrm{P}}=$ Proportional gain

$\mathrm{e}=$ error

$\mathrm{t}=$ instantaneous (present) time

\section{b. Integral Response}

The Integral component sums the error term over time. The result is that even a small error term will cause the integral component to increase slowly. The integral term in a PID controller is the sum of the instantaneous error over time and gives the accumulated offset that should have been corrected previously. The accumulated error is then multiplied by the integral gain $\left(\mathrm{K}_{\mathrm{I}}\right)$ and added to the controller output.

The integral response is given by:

$\mathrm{I}_{\text {out }}=\mathrm{K}_{\mathrm{I}} \int_{0}^{t} \mathrm{e}(\tau) \mathrm{d} \tau$

Where, $\mathrm{K}_{\mathrm{I}}=$ Integral gain

$\tau=$ Variable of integration, takes on values from time 0 to present time, $\mathrm{t}$.

\section{c. Derivative Response}

The Derivative response in a PID controller is calculated by determining the slope of the error over time and multiplying this rate of change by the derivative gain $\left(\mathrm{K}_{\mathrm{D}}\right)$.

The derivative response is given by:

$\mathrm{D}_{\text {out }}=\mathrm{K}_{\mathrm{D}}\left(\frac{d(e(t))}{d t}\right)$ 
Where, $\mathrm{K}_{\mathrm{D}}=$ derivative gain

\section{PID controller transfer function}

The controller output, $u(t)$,combining equations (1), (2) and (3) is given by:

$\mathrm{u}(\mathrm{t})=\mathrm{K}_{\mathrm{p}} * \mathrm{e}(\mathrm{t})+\mathrm{K}_{\mathrm{I}} \int_{0}^{\mathrm{t}} \mathrm{e}(\tau) \mathrm{d} \tau+\mathrm{K}_{\mathrm{D}}\left(\frac{d(e(t))}{d t}\right)$

In continuous s-domain, the corresponding PID controller transfer function, $\mathrm{G}_{\mathrm{C}}(\mathrm{s})$ is given by [3]:

$\mathrm{G}_{\mathrm{C}}(\mathrm{s})=\mathrm{K}_{\mathrm{P}}+\mathrm{K}_{\mathrm{I}} / \mathrm{s}+\mathrm{K}_{\mathrm{D}}{ }^{*} \mathrm{~s}$

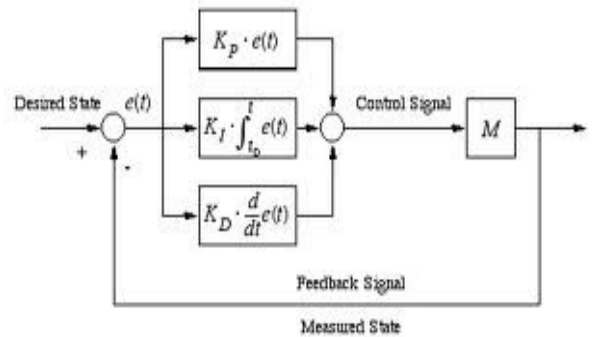

Figure 1. General block diagram of PID controller

\section{Application of PID control in mobile robotics}

Proportional-Integral-Derivative (PID) control is one of the most common control algorithms used in automation sector and has been universally accepted in industrial control in spite of the development of advanced control strategies [4]. The popularity of PID controllers can be attributed partly to their robust performance in a wide range of operating conditions and partly to their functional simplicity, which allows engineers to operate them in a simple, straightforward manner. PID controllers are widely used in the robotic industry in controlling the operation of mobile robots.

Mobile robotics is a field of great interest and rapid development. With related technologies changing rapidly, different kinds of mobile robots have been widely used in many indoor and outdoor applications such as spot welding, loading/unloading of parcels, vacuum cleaning, defence and security [5]. In this paper, we are dealing with the navigation of mobile robot namely, the Khepera 3and iRobot Create using PID controller.

The Khepera 3 robot integration is a product of the Swiss company-K Team. Features available on the platform include an upgradable embedded computing power using the KoreBot system, multiple sensor arrays for both long range and short range object detection, swappable battery pack system for optimal autonomy, and differential drive odometry[6]. The Khepera 3 robot embeds lots of sensors and advanced computational power which enhances its application in education and research.

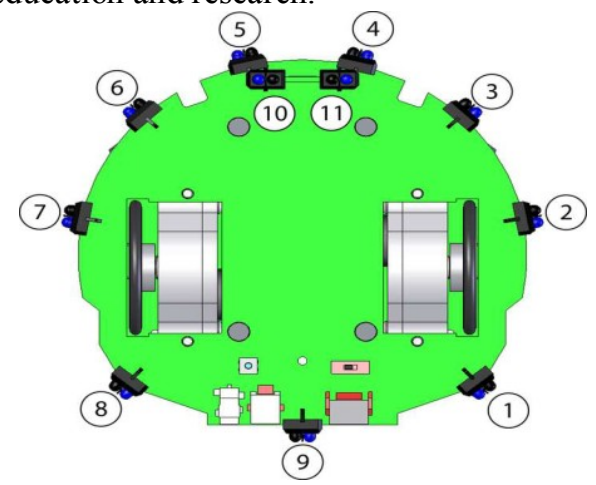

Figure 2: Position of the IR sensors in Khepera 3

Khepera 3 is equipped with 11 infrared (IR) range sensors, of which nine are located in a ring around it and two are located on the underside of the robot. The IR sensors are complemented by a set of five ultrasonic sensors. It is powered by a single battery on the underside and can be controlled via software on its embedded Linux computer. 


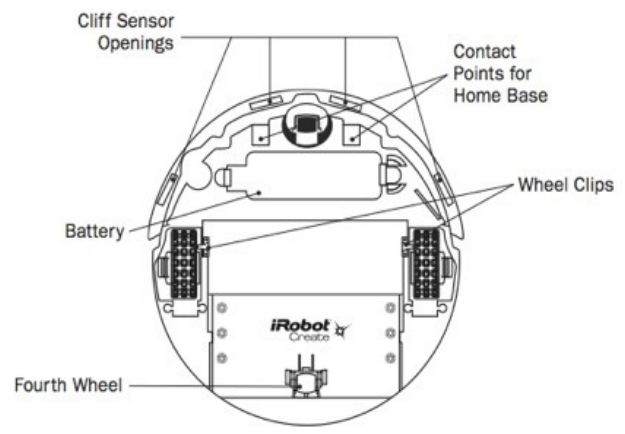

Figure 3: Position of wheel and sensors in iRobot Create

Create is a mobile robot platform developed by the firm-iRobot which is used by students, educators and developers. It is a preassembled robot. Users can write custom software using a variety of methods that apply "streaming sensor data" mode of the robot for more control. [7]

The subsequent parts of the paper discuss the various control algorithms designed and tested on these robotic platforms with their related results. Simulation of the algorithms was done on the Khepera 3 robot and a realtime implementation was carried out on the iRobot Create robotic platform. Figure 4 clearly depicts the control flow of the algorithms used.

\section{Go to angle $\longrightarrow$ Go to goal $\Longrightarrow$ Avoid obstacle}

Figure 4: Steps for building the various algorithms based on the PID control.

\section{Go To Angle}

Go To Angle steers the robot towards a angle with a constant velocity using PID controller. This can be done using MATLAB program with the following steps:

Step 1: Compute the left and right wheel speeds for go to angle.

Step 2: Inputs are given to the robot ('theta the desired angle in radians and ' $v$ 'the linear velocity in meter/second.)

Step 3: Output variables are defined. ('v' and 'w', 'v' is the linear velocity and 'w' is angular velocity).

Step 4: Gains are specified. $\left(K_{P}, K_{I}\right.$ and $\left.K_{D}\right)$

Step 5: Go To Angle algorithm is developed.

Step 6: Error tracking is done by continuously calculating the error difference between the desired angle 'theta_d' and the angle calculated from the sensor data input 'theta'. The robot will continue this process until the error becomes zero thus maintaining the angle.

For error tracking we use atan 2 command to keep the error in the range $[-\pi, \pi][8]$.

Step 7: Result is achieved.

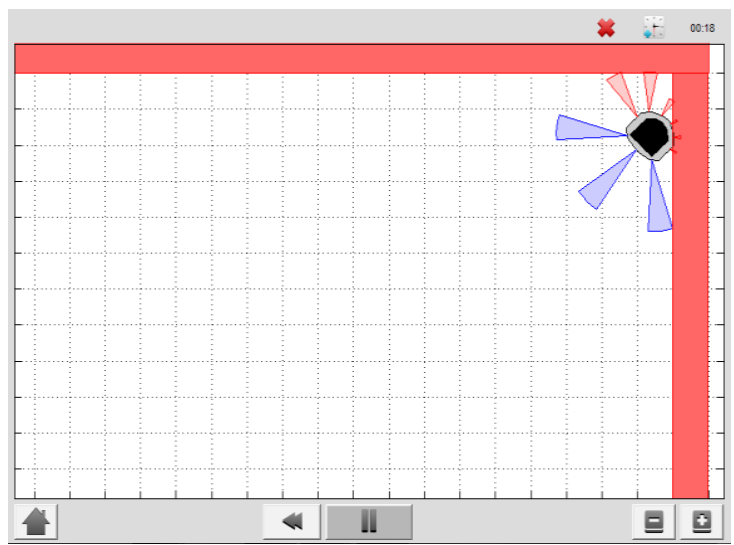

Figure 5: Go To Angle.

Since there is no distance specified or any obstacle avoidance code developed the robot will continue to move even after the desired angle is achieved (theta-d - theta $=0$ ). Thus when the robot reaches the red wall it will crash and stop at that position [8]. 

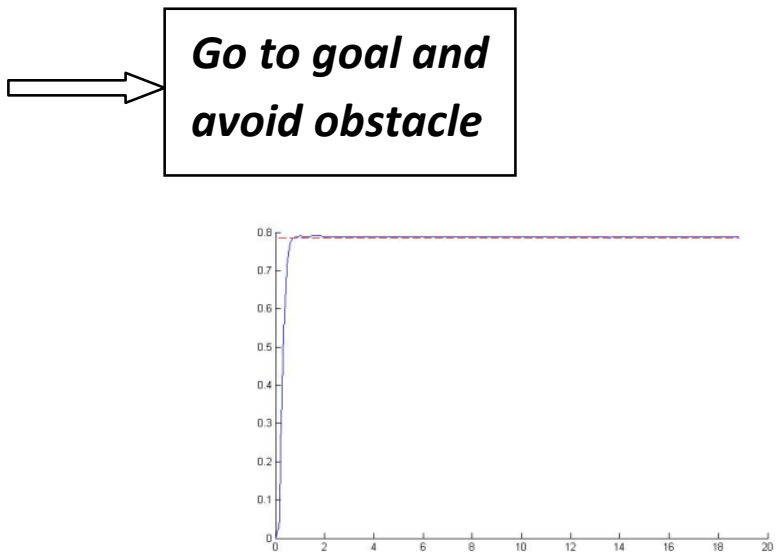

Figure 6: Go To Angle output curve

Figure 6 is a go to angle output curve which shows change in angle with respect to time in the blue line, the red dotted line shows the desired angle. The curve shows very smooth change in angle.

\section{Go To Goal}

The objective behind Go To Goal in a MATLAB programmed Khepera 3 robot is to steer the robot towards that goal in a constant velocity using PID controller. The steps are as follows: Step1: Define the input

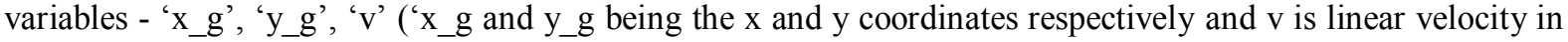
meter/second). Step 2: Define the output variables which the PID controller needs to control. They are ' $v$ ' and ' $\mathrm{w}$ ' ( $\mathrm{v}$ and $\mathrm{w}$ are linear and angular velocities respectively).

Step 3: Specify the gains $K_{P}, K_{I}$ and $K_{D}$ initially for the go to goal operation which are calculated experimentally. Step 4: Initialize the error values with 0 which will change with the linear and angular movement of the robot. Step 5: Extract the values of linear distance ' $\mathrm{X}$ ', ' $\mathrm{Y}$ ' and the angle 'theta' of the robot from the initial starting position. Step 6: Calculate the Proportional, Integral, and Derivative terms for the PID regulator that steers the robot to the goal. The PID regulator needs three parts to be implemented:

(a)The first part is the proportional term e e P. It is simply the current error $\mathrm{e}_{-} \mathrm{k}$. $\mathrm{e}_{-} \mathrm{P}$ is multiplied by the proportional gain $\mathrm{Kp}$ when computing $\mathrm{w}$. (b) The second part is the integral term $\mathrm{e}_{-}^{-}$. The integral needs to be approximated in discrete time using the total accumulated error $\mathrm{E}_{-} \mathrm{k}$, the current error e $\mathrm{k}$, and the time step dt. e $\mathrm{I}$ is multiplied by the integral gain $\mathrm{K}_{\mathrm{I}}$ when computing $\mathrm{w}$, and is also saved as $\mathrm{E}_{-} \mathrm{k}$ for the next time step. (c) The third part is the derivative term e D. The derivative needs to be approximated in discrete time using the current error $\mathrm{e}_{-} \mathrm{k}$, the previous error $\mathrm{e}_{-} \mathrm{k}_{-}$, and the the time step dt. $\mathrm{e}_{-} \mathrm{D}$ is multiplied by the derivative gain $K_{D}$ when computing $w$, and the current error $e_{-} k$ is saved as the previous error $e_{-} k_{-} 1$ for the next time step [9].

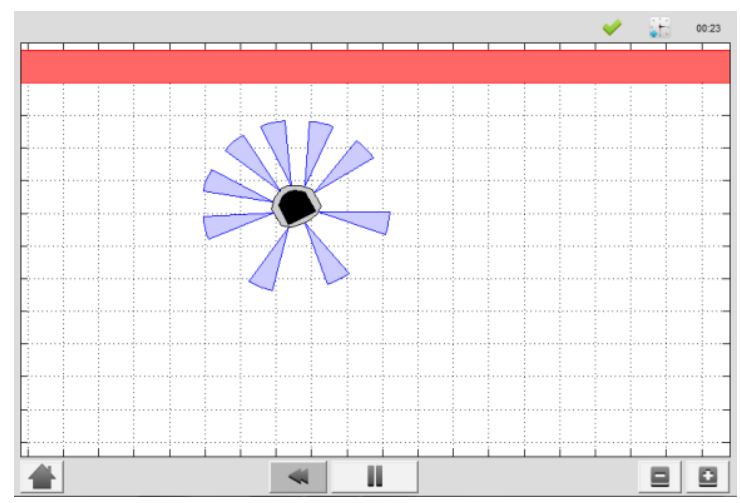

Figure 7: Go To Goal. 


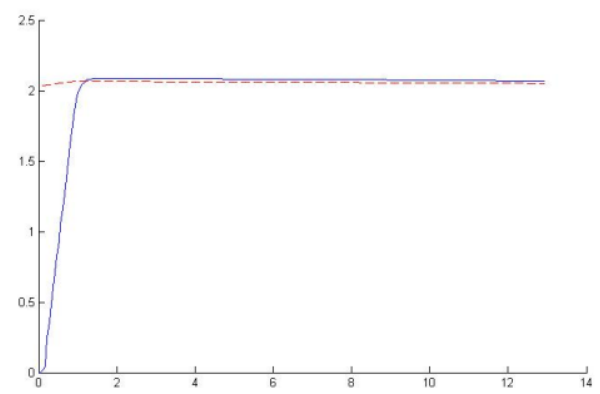

Figure 8: Go to Goal output curve

The output curve shows change in angle with respect to time in the blue line, the red dotted line shows the desired angle. The curve shows very smooth change in angle and also the goal is reached in a very short span of time.

\section{Avoid Obstacle}

The objective is implementing the different parts of a controller that steers the robot successfully away from obstacle to avoid a collision. This is known as the avoid-obstacle behavior. The IR sensors allow us to measure the distance to obstacles in the environment, but we need to compute the points in the world to which these distances correspond.

To perform this, the steps required are:-

Step 1: Transform the IR distances to points in the world.

Step 2: Compute a vector to each point from the robot, $\mathrm{u}_{1}, \mathrm{u}_{2}, \mathrm{u}_{3, \ldots . .,} \mathrm{u}_{9}$.

Step 3: Weigh each vector according to their importance, $\mathrm{a}_{1} \mathrm{u}_{1}, \mathrm{a}_{2} \mathrm{u}_{2}, \mathrm{a}_{3} \mathrm{u}_{3, \ldots}, \mathrm{a}_{9} \mathrm{u}_{9}$. For example, the front and side sensors are typically more important for obstacle avoidance while moving forward.

Step 4: Sum the weighted vectors to form a single vector, $u_{0}=a_{1} u_{1+} a_{2} u_{2}+a_{3} u_{3+\ldots}+a_{9} u_{9}$

Step 5: Use this vector to compute a heading and steer the robot to this angle.

The $\mathrm{K}_{\mathrm{P}}, \mathrm{K}_{\mathrm{I}}, \mathrm{K}_{\mathrm{D}}$ values are kept same for calculating the change in angular velocity ' $\mathrm{w}$ ' in radian/second which will steer the robot away from the obstacle [9].

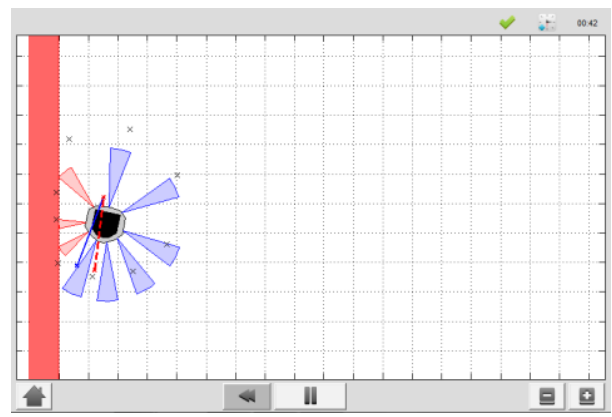

Figure 9: Avoid Obstacle (side wall)

In Figures 9 and 10 the robot reaches the red wall where the IR sensors sense an obstacle either on the front sensors or the back sensors (sensors 2 to 7 in the Khepera 3 are weighed more than sensors 1,8,9). This causes the vector $\mathrm{u}_{0}$ shown by red dotted line change the direction to steer the robot away from the obstacle.

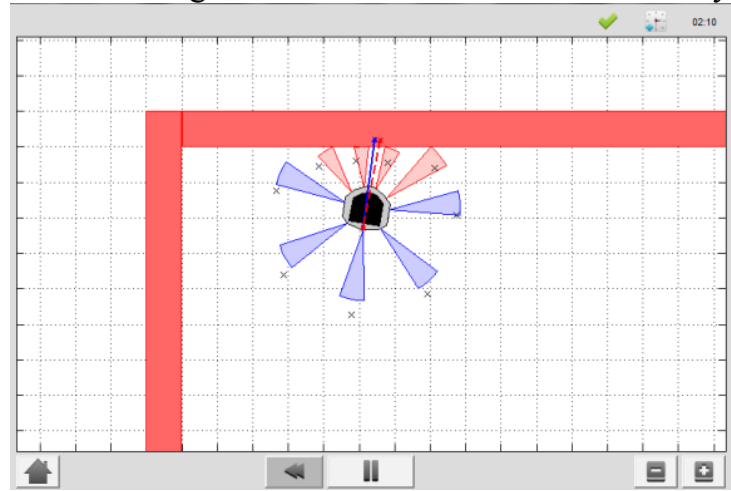

Figure 10: Avoid Obstacle (top wall) 


\section{a. Blending}

\section{Go To Goal and Avoid Obstacle}

Blending provides us to make a small improvement to the go-to-goal and avoid-obstacle controllers. Arbitration between the two controllers allows the robot to drive to a goal, while not colliding with any obstacle on the way. We will improve the performance of both the Go-to-Goal and Avoid-Obstacle behavior by dynamically adjusting the linear velocity based on the angular velocity of the robot. The actuator limits of the robot limit the linear velocity to a range of $[-0.3 ; 0.3] \mathrm{m} / \mathrm{s}$ and the angular velocity to a range of $[-2.765$; $2.765] \mathrm{rad} / \mathrm{s}$. It is important to remember that with a differential drive, we cannot, drive the robot at the maximum linear and angular velocities. There is a trade-off between linear and angular velocities: linear velocity has to decrease for angular velocity to increase, and vice versa. So, we have designed the system accordingly. Now we start the blending process. The solutions to the Go-to-Goal and Avoid-Obstacle controllers have been combined into a single controller, one vector pointing to the goal from the robot, and another vector pointing from the robot to a point in space away from obstacle. These two vectors are combined (blended) into a component vector, which is a vector that points the robot both away from obstacle and towards the goal [9].

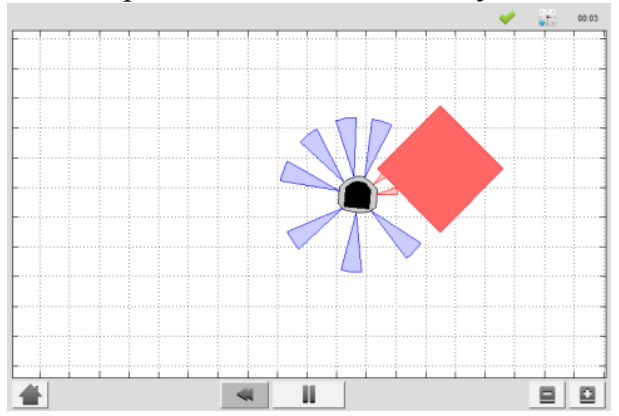

Figure 11: Blending (avoid obstacle)

Figure 11 shows the process of blending. Here the robot tries to avoid the obstacle and reach its goal.

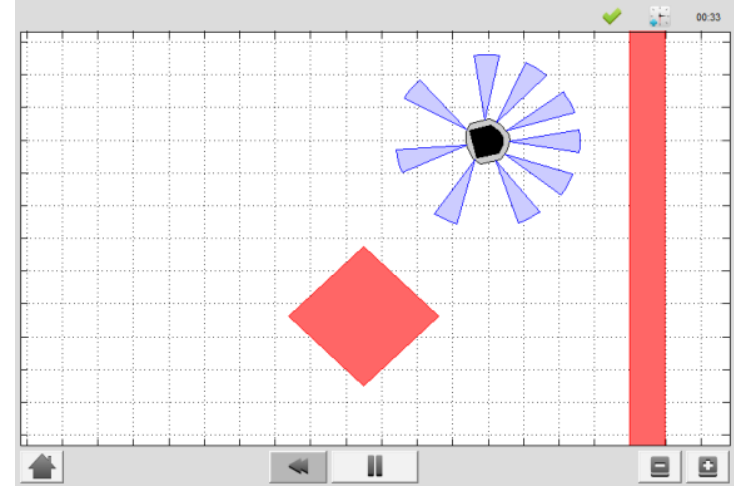

Figure 12: Blending (go to goal)

In Figure 12, the robot has reached its goal by avoiding the obstacle.

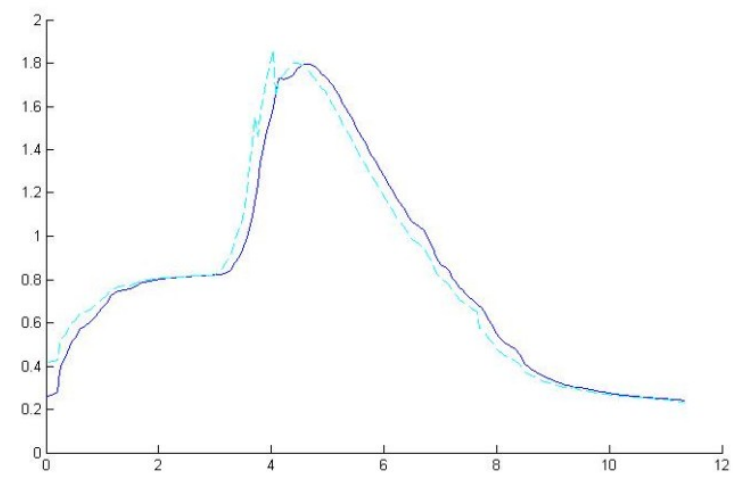

Figure 13: Blending output curve

In Figure 13, the graph shows us the output curve of blending. The robot successfully navigates to the fixed goal location without colliding with the obstacle that is in the way. Once the robot is near the goal, it stops. 
The blending controller's advantage is that it smoothly blends Go-to-Goal and Avoid - Obstacle together. However, when there are no obstacle around, it is better to purely use go-to-goal, and when the robot gets dangerously close, it is better to only use avoid-obstacle. The switching

logic performs better in those kinds of situations.

\section{b. Hard switching}

In Hard switching logic the robot continuously switches between its two objectives i.e., Go to Goal and Avoid - Obstacle, while it tries to avoid an obstacle before reaching the goal.

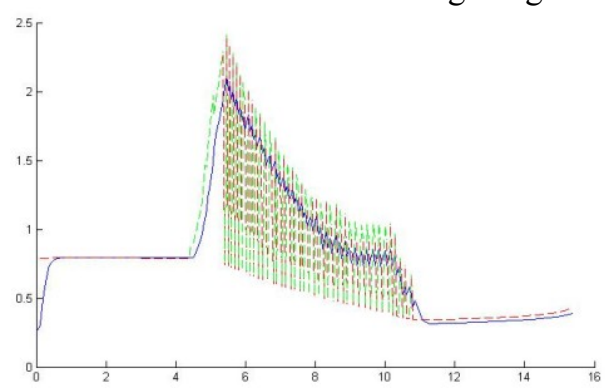

Figure 14: Hard switching output curve

The Hard switching technique has greater efficiency in performing the objective (avoid obstacle and go to goal) at higher linear velocities. But as the output curve has depicted, the switching technique causes heavy vibrations in the robot, which can cause damage to the internal hardware of the Khepera 3 or other robots. This makes blending technique more acceptable as it uses a priority based technique. When the priority is more to avoid obstacle the robot will perform only avoid obstacle objective, otherwise it will perform go to goal.

This process causes much smoother transition between the Go to Goal and Avoid - Obstacle.

\section{Go In a Rectangle using iRobot Create}

For practically testing the objectives in real-time, the Go To Goal objective is done on iRobot Create. The objective is to steer the robot such that it moves in a rectangular path. The robot must stop after traveling the entire perimeter. Step 1: To travel length forward, create the distance entered in meters. Positive distances move the Create forward, negative distances move the Create backwards. Speed should be between 0.025 and $0.5 \mathrm{~m} / \mathrm{s}$. Step 2: To turn angle specify an angle to turn though (in degrees). Positive is counter clockwise and negative is clockwise and a speed (in $\mathrm{rad} / \mathrm{sec}$ ). Robot turns in place and stops when done. Speed is in $\mathrm{rad} / \mathrm{sec}$ (between 0 and 0.2).For the first turn 90 degree angle is given. Step 3: To travel the breadth forward create forward distance in meter. Speed should be between 0.025 and $0.5 \mathrm{~m} / \mathrm{s}$. Step 4: Turn angle by +90 degree. Step 5: Travel length forward. Step 6: Turn angle by +90 degree. Step 7: Travel breadth forward. Following this procedure the iRobot Create can travel a rectangular path using PID control.

\section{Discussions and Conclusion}

A PID controlled navigation system for mobile robots, Khepera 3 and iRobot Create is implemented using MATLAB. The proposed algorithm optimised parameters of PID controller which is applied to the simulation and practical experimentation schemes.

In case of the navigation system of Khepera 3, for Go To Angle task, only Proportional control is used. For Go to Goal task, small Integral and Derivative control is applied along with Proportional control. In the comprehensive navigation system combining the tasks of Go To Angle, Go To Goal and Avoid Obstacle, there is a compromise weight to be assigned to Go To Goal and Avoid Obstacle tasks respectively depending upon the environment in which the robot is navigating. Blending and Hard-switching are the methods used for combination of the tasks. While Blending ensures smoother navigation minimising vibrations in the robot, Hardswitching is a more efficient technique when the linear velocity of the robot is high.

In case of the navigation system of iRobot Create, the robot traverses a rectangular path in counter clockwise direction using adequate Proportional, Integral and Derivative control.

The final results show that the proposed control system for both the robots respectively, successfully provides a simple and effective method of mobile robot navigation.

Future work will include developing algorithm for Avoid Obstacle and combination of Go to Angle, Go To Goal and Avoid Obstacle on iRobot Create platform. Also tracking of reference trajectory by optimising PID parameters using genetic algorithm can be implemented on Khepera 3 platform in future. 


\section{Acknowledgement}

The authors are grateful to ESL (www.eschoollearning.net), Kolkata for providing hardware and software support in carrying out this work. The authors would also like to extend their gratitude towards the organization for the detailed intellectual support provided to them during the project.

\section{References}

[1]. "PID Controller" [Online]. Available: http://en.wikipedia.org/wiki/PID controller.

[2]. "PID theory" [Online]. Available: http://www.ni.com/white-paper/3782/en/

[3]. RAJKUMAR BANSAL, A.PATRA, VIJAYBHURIA, "Design of PID Controller for Plant Control and Comparison with Z-N PID Controller", International Journal of Emerging Technology and Advanced Engineering, Volume 2, Issue 4, April 2012.

[4]. S. N. DEEPA, G. SUGUMARAN, "Design of PID Controller for Higher Order Continuous Systems using MPSO based Model Formulation Technique", International Journal of Electrical and Electronics Engineering, 5:4 2011.

[5]. CHIA-JU WU, TING-LI CHIEN, TSONG-LI LEE, and LI-CHUN LAI, "Navigation of a mobile robot in indoor environment", Journal of the Chinese Institute of Engineers, Vol. 28, No. 6, pp. 915-924 (2005).

[6]. "Khepera 3" [Online]. Available: http://www.k-team.com/mobile-robotics-products/khepera-iii.

[7]. "iRobot Create" [Online]. Available: http://www.irobot.com/us/learn/Educators/Create.aspx.

[8]. "Simiam"[Online]. Available: http://jdelacroix.github.io/simiam/.

[9]. Sim.I.am: A Robot Simulator by Jean-Pierre de la Croix. 